\title{
A Simulation Model for Nakagmi-m Fading Channel with $m>1$
}

\author{
Sandeep Sharma \\ School of ICT \\ Gautam Buddha University \\ Greater Noida, India
}

\author{
Rajesh Mishra \\ School of ICT \\ Gautam Buddha University \\ Greater Noida, India
}

\begin{abstract}
In this paper, we propose a model to simulate a wireless fading channel based on Nakagami-m distribution with $m>1$. The Nakagami-m fading channel is the most generalized distribution as it can generate one-sided Gaussian distribution, Rayleigh distribution and Rician distribution for $m$ equals to 0.5 , 1 and $>1$ respectively. In this work we have proposed a method to generate a wireless fading channel based on Nakagami-m distribution as this distribution fits to a wide class of fading channel conditions. Simulation results were obtained using Matlab R2013a and compared with the analytical results.
\end{abstract}

Keywords-Nakagami Distribution; Fading Channel; Wireless Channel Modeling

\section{INTRODUCTION}

In wireless communication fading plays a vital role in the channel estimation. Fading is the rapid fluctuation in the received signal strength of the wireless signal. Communication systems are subjected to fading caused by multipath propagation due to reflections by surrounding objects, refractions and scattering by buildings and other large structures. Thus, the received signal is a sum of various signals that arrive at the receiver via different propagation paths which may be direct line of sight (LOS) or non line of sight path (non-LOS). To model fading in wireless communication, several techniques have been used in literature. As the nature of the wireless channel is random, it has to be model statistically. Several statistical models have been used in the literature to describe the fading envelope of the received signal [6],[13]-[15].

The Rayleigh and Rician distributions are used to characterize the fading envelope of the wireless signals over small geographical areas or short term fades while the lognormal distribution is used when much wider geographical areas are involved. A more versatile statistical model, however, is Nakagami's m-distribution [1], which can model a variety of fading environments including those modeled by the Rayleigh and one-sided Gaussian distributions. Also the log-normal and Rician distributions may be closely approximated by the Nakagami distribution in some ranges of mean signal values [16]. The fit between Nakagami and Rician distributions is very accurate for low signal-to-noise ratio (SNR) values in comparison to large SNR values. Furthermore, the Nakagami distribution is more flexible and more accurately fit experimental data for many physical propagation channels then the log-normal and Rician distributions [16],[17]. We may find various research papers where Nakagami distribution is used to simulate in applications like satellite communication, vehicular to vehicular communication, even it is applied in medical applications such as ECG and ultrasound signals. Although the Nakagami model fits experimental data around the mean or median, but it is reported in [18] that it does not fit very well in the tails of the distribution. In spite this, the Nakagami distribution is much popular and used by many of the researchers in their domain whether it may be wireless, medical, terrestrial signal analysis, and vehicular ad-hoc networks.

Paper Organization: This paper is organized as follows. In section II, we discussed the theoretical background of wireless channel modeling and the factors affecting it. Section III explains the Nakagami-m distribution followed by the simulation method in section IV. Section V discusses the various results and their analysis. Finally, section VI.

\section{THEORITICAL BACKGROUND}

In this section, we explain theoretical background of a wireless channel and the factors affecting the channel response. A wireless channel is different from a wired channel as it contains multipath components from direct line of sight (LOS) component and various reflected and refracted components. The wireless channel is made by the constructive and destructive addition of different multipath components introduced by the channel. The same phase components are added and the out of phase components are subtracted and their algebraic sum is what we get at the antenna of the receiver. In general, the deterministic channel models are rarely available as the nature of the channel is random, and thus we need to characterize multipath channels statistically. If a single pulse is transmitted over a multipath channel, then the received signal will not be a single pulse but appear as a series of pulses, with each pulse in the series corresponds to the LOS component or an individual multipath component associated with a discrete scatterer or cluster of scatterers. The channel characteristics certainly depends upon the number of scatterer objects, number of multipath, size of the objects and the amount of absorption by the surrounding environment such as wall and roofs (thickness and material has a impact on the degree of absorption). Another characteristic of the multipath channel is its time-varying nature. This variation in time arises because either the transmitter or the receiver is moving, and this mobility of the transmitter and/ or receiver therefore change the location of reflectors in the transmission path, which give rise to multipath, will change over time. 


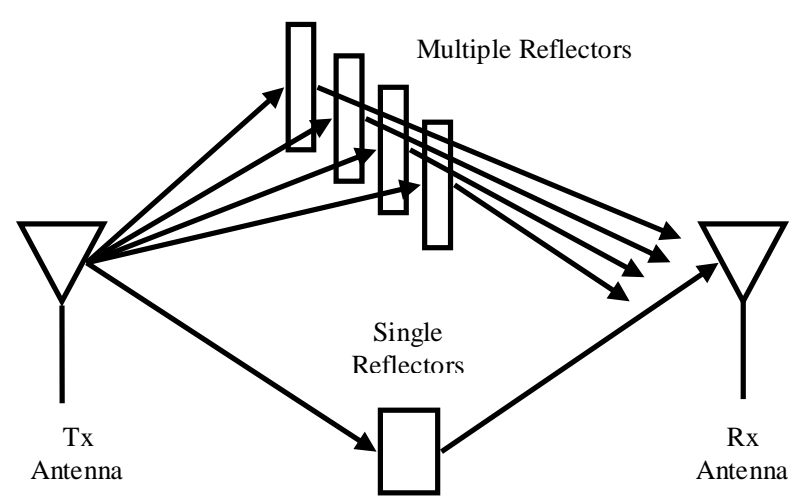

Fig. 1. Multipath due to single and multiple reflectors

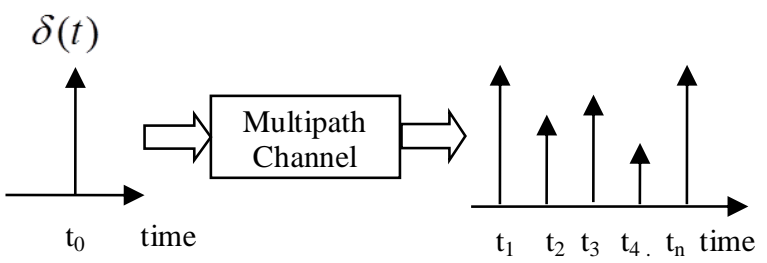

Fig. 2. Effect of Multipath when only a single impulse is transmitted

Another important characteristic of a multipath channel is the time delay spread which is caused to the received signal. This delay spread equals the time of arrival of the first received signal component (LOS or multipath) and the last received signal component associated with a single transmitted pulse. When the delay spread is small compared to the inverse of the signal transmission bandwidth then there is little time spreading in the received signal. However, when the delay spread is relatively large, there is considerable time spreading of the received signal which can lead to signal distortion substantially.

\section{A. Envelope and Power Distribution in the Signal}

Consider any two Gaussian random variables $\mathrm{X}$ and $\mathrm{Y}$, both with zero mean and equal variance $\sigma 2$, then resultant $R=\sqrt{X^{2}+Y^{2}}$ of $\mathrm{X}$ and $\mathrm{Y}$ can be related by Rayleigh-distributed and $\mathrm{Z} 2$ is exponentially distributed. If $r_{I}$ and $r_{Q}$ represents the in-phase and quadrature phase components having the variance of $\sigma 2$, then the envelope of the signal is given by

$$
z(t)=|r(t)|=\sqrt{r_{I}^{2}+r_{Q}^{2}}
$$

and it is Rayleigh distributed by the well known distribution given by

$$
p_{Z}(z)=\frac{2 z}{P_{r}} \exp \left[-\frac{z^{2}}{P_{r}}\right]=\frac{z}{\sigma^{2}} \exp \left[-\frac{z^{2}}{2 \sigma^{2}}\right], x \geq 0,
$$

where, $P_{r}=\sum_{n} E\left[\alpha_{n}^{2}\right]=2 \sigma^{2}$ is the average received power based on the path loss and shadowing alone. Making substitution $z^{2}(t)=|r(t)|^{2}$ in the Rayleigh distribution we get:

$$
p_{Z^{2}}(x)=\frac{1}{P_{r}} e^{-\left(\frac{x}{P_{r}}\right)}=\frac{1}{2 \sigma^{2}} e^{-\left(\frac{x}{2 \sigma^{2}}\right)}, \quad x \geq 0
$$

This shows that the received power is exponentially distributed over mean $2 \sigma^{2}$. So $r(t)$ has a Rayleigh-distributed amplitude and uniform phase, and the two are mutually independent. When the channel has a fixed line of sight (LOS) component, then $r_{I}(t)$ and $r_{Q}(t)$ do not have zero-mean. In such a case, the received signal is the superposition of a complex Gaussian component and a LOS component. The signal envelope in this case can be shown to have a Rician distribution and given by:

$$
p_{Z}(z)=\frac{z}{\sigma^{2}} \exp \left[\frac{-\left(z^{2}+s^{2}\right)}{2 \sigma^{2}}\right] I_{0}\left(\frac{z s}{\sigma^{2}}\right), \quad x \geq 0,
$$

where, $2 \sigma^{2}=\sum_{n, n \neq 0} E\left[\alpha_{n}^{2}\right]$ is the average amount of power in the non-line of sight component and $s^{2}=\alpha_{0}^{2}$ is the average amount of power in the line of sight (LOS) component of the radio signal. Here $\mathrm{I}_{0}$ is the modified Bessel's function of zero order. The Rician fading has the average received power which is given by the equation:

$$
P_{r}=\int_{0}^{\infty} z^{2} p z(z) d x=s^{2}+2 \sigma^{2}
$$

Very often, the Rice distribution is described in terms of fading parameter " $K$ " defined as

$K=\frac{s^{2}}{2 \sigma^{2}}=\frac{\text { Power in the LOS component }}{\text { Power in thenon - LOS multipath component }}$

Different values of $\mathrm{K}$ give us different fading statistics and thus it is a factor that controls the amount of fading in the wireless channel. For $\mathrm{K}=0$ we have Rayleigh fading, and for $\mathrm{K}=1$ we have no fading, i.e. a channel with no multipath and we have a LOS component. The fading parameter " $\mathrm{K}$ " is therefore a measure of the severity of the fading: a small " $\mathrm{K}$ " implies severe fading, a large value of $\mathrm{K}$ implies low fading.

$$
K=\left\{\begin{array}{rr}
0 & \text { Rayleign Fading } \\
1 & \text { No Fading only LOS }
\end{array}\right.
$$

If we substitute $s^{2}=K P / K+1$ and $2 \sigma^{2}=P / K+1$ the Rician distribution can be obtain in terms of $\mathrm{K}$

$$
p_{Z}(z)=\frac{2 z(K+1)}{P_{r}} \exp \left[-K-\frac{(K+1) z^{2}}{P_{r}}\right] I_{0}\left(2 z \sqrt{\frac{K(K+1)}{P_{r}}}\right), z \geq 0
$$

The more general distribution is the Nakagami distribution with the help of which we can generate both the Rayleigh fading as well as Rician fading.

\section{The NAKAgami Distribution}

With Nakagami-m distribution [1], usually denoted by mdistribution, a wide range of fading channel conditions can be modeled. 
This fading distribution has often provides the best fit to land-mobile, indoor mobile multipath propagation as well as for the ionospheric radio links [2]. Recent studies also showed that Nakagami-m gives the best fit for satellite-to-indoor and satellite-to-outdoor radio wave propagation as well $[3,4]$. The channel response of a wireless channel is a complex quantity and for this reason let us assumes that the complex valued Nakagami-m fading channel $\mathrm{Z}$ is represented as follows:

$$
Z=X+j Y=\mathrm{R}\left(e^{j \Theta}\right)
$$

where $\mathrm{X}, \mathrm{Y}, \mathrm{R}$ and $\Theta$ represents the in-phase component, the quadrature-phase component, the envelope and the phase component simultaneously. The probability density function (PDF) for a Nakagami-m distributed fading envelope R can be expressed as:

$$
f_{R}(r)=\frac{2 m^{m} r^{2 m-1}}{\Gamma(m) \Omega^{m}} \exp \left(-\frac{m r^{2}}{\Omega}\right), \quad 0 \leq r<\infty
$$

Where, $\Omega=E[R 2]$, is the expected value of the average power and $\mathrm{m}$ is shaping parameter which controls the shape of the distribution. When $\mathrm{m}$ is integer, $\mathrm{R}$ is the square root of the sum of the amplitude square of mi.i.d. complex GaussianRVs,

$$
R=\sqrt{\left|x_{1}^{2}\right|+\left|x_{2}^{2}\right|+\left|x_{3}^{2}\right|+\cdots\left|x_{m}^{2}\right|}
$$

Where $\mathrm{x}_{\mathrm{i}}=1,2,3 \ldots \ldots \mathrm{m}$ is a complex Gaussian random variable (RV) with zero mean and variance $\Omega / \mathrm{m}$. Here $E($.$) is$ the expectation operator and $\Gamma$ (.) is the gamma function. $m$ is the inverse of the normalized variance of $\mathrm{R} 2$ :

$$
m=\frac{\left(E\left[R^{2}\right]\right)^{2}}{\operatorname{Var}\left(R^{2}\right)}=\frac{\Omega^{2}}{\operatorname{Var}\left(R^{2}\right)}
$$

where $\operatorname{Var}\left(R^{2}\right)$ is the variance of $R^{2}$.The value for $\mathrm{m}$ ranges between $1 / 2$ and $\infty$. When $m \rightarrow \infty$, the channel converges a static channel i.e. it no longer remains variant channel.[5]. As special cases, for $m=1$ the Nakagami-m become Rayleigh distribution one-sided Gaussian distribution for $m=1 / 2$. This principally means that, fading is more severe than Rayleigh fading if $\mathrm{m}<1$, and for values of $\mathrm{m}>1$, the fading is less severe. For the values of $\mathrm{m}>1$, the Nakagami-m distribution closely approximates the Rician distribution. The Nakagami shape parameter $m$ and the Rician factor $\mathrm{K}$ which determines the severity of fading in case of the Rician fading can be related to the following equation [5]:

$$
\begin{aligned}
& m=\frac{(K+1)^{2}}{2 K+1}, \text { for } \mathrm{K} \geq 0
\end{aligned}
$$

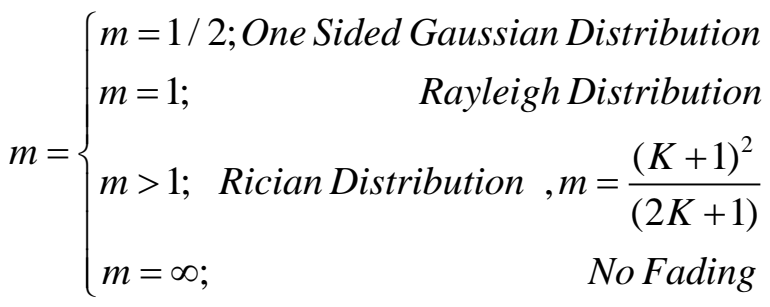
by

The Nakagami-m phase envelope joint distribution is given

$$
f_{R \Theta}(r, \theta)=\frac{m^{m}|\sin (2 \theta)|^{m-1} r^{2 m-1}}{2^{m-1} \Omega^{m} \Gamma^{2}\left(\frac{m}{2}\right)} \exp \left(-\frac{m r^{2}}{\Omega}\right)
$$

The envelope pdf $f_{R}(r)$ is given by the well known formula

$$
f_{R}(r)=\frac{2 m^{m} r^{2 m-1}}{\Gamma(m) \Omega^{m}} \exp \left(-\frac{m r^{2}}{\Omega}\right), 0 \leq r<\infty
$$

And the phase envelope is given by:

$$
\begin{gathered}
f_{\Theta}(\theta)=\frac{\Gamma(m)|\operatorname{Sin} 2 \theta|^{m-1}}{2^{m} \Gamma^{2}(m / 2)}, \quad-\pi \leq \theta<\pi \\
f_{X}(u)=f_{Y}(u)=\frac{m^{(m / 2)}|u|^{m-1}}{\Omega^{(m / 2)} \Gamma(m / 2)} \exp \left(-\frac{m u^{2}}{\Omega}\right),-\infty<u<\infty
\end{gathered}
$$

\section{Simulation Method}

A pair of correlated Nakagami fading envelopes can be generated keeping the integer value of the shaping or fading parameter, from m-dimensional i.i.d. (independent and identically distributed) complex Gaussian distributed column vector $\bar{Z}_{1}$ and $\bar{Z}_{2}$ i.e.

$$
\begin{gathered}
\bar{Z}_{1}=\left[\begin{array}{lllll}
x_{1} & x_{2} & x_{3} & \ldots & x_{m}
\end{array}\right]^{T} \text { and } \bar{V}_{1}=\left|\bar{Z}_{1}\right|^{2} \\
\bar{Z}_{2}=\left[\begin{array}{lllll}
y_{1} & y_{2} & y_{3} & \ldots & y_{m}
\end{array}\right]^{T} \text { and } \bar{V}_{1}=\left|\bar{Z}_{1}\right|^{2} \\
x_{i}=x_{\text {ireal }}+x_{\text {iim }} \text { and } y_{i}=y_{\text {ireal }}+y_{\text {iim }}
\end{gathered}
$$

where $x_{1}, x_{2} \cdots x_{m}$ and $y_{1}, y_{2} \cdots y_{m}$ are complex Gaussian $\mathrm{RVs}$ with zero mean and variance $\sigma_{x}^{2}$ and $\sigma_{y}^{2}$ respectively. We assume that the power in the real part and imaginary part be equal in magnitude. Here, $x_{\text {ireal }}, y_{\text {ireal }}, x_{\text {iim }}, y_{\text {iim }}$ have normalized envelope correlation coefficients $\rho_{x y}$ and its range lies between 0 and 1, i.e. $\left(0 \leq\left|\rho_{x y}\right| \leq 1\right)$ and at the same time the real and imaginary parts of the random variables $x_{i}$ and $y_{i}$ remains uncorrelated. In eqn 18 and eqn 19, transpose is denoted by T. Here, $\rho$ is the correlation of power between $\bar{V}_{1}$ and $\bar{V}_{2}$ and related as follows:

$$
\rho=\left|\rho_{x y}\right|^{2}=\frac{\operatorname{cov}\left(\bar{V}_{1}, \bar{V}_{2}\right)}{\sqrt{\operatorname{var}\left(\bar{V}_{1}\right) \cdot \operatorname{var}\left(\bar{V}_{2}\right)}}
$$

Where, $\operatorname{var}\left(\bar{V}_{1}\right)=\Omega, \operatorname{var}\left(\bar{V}_{1}\right)=\widehat{\Omega}$ and $(0 \leq \rho \leq 1)$. The joint pdf of $\sqrt{\bar{V}_{1}}$ and $\sqrt{\bar{V}_{2}}$ gives the joint pdf of the nakagami-m distribution. 
The following steps are involved in the generation of the Nakagmi-m fading channel

Step1: First generate $m$ i.i.d. Gaussian random variables.

Step2: Generate vectors $\bar{Z}_{1}$ and $\bar{Z}_{2}$ using eqn 18 and eqn 19.

Step3: Calculate var, cov and $\rho$ using eqn 21.

Step4: Generate complex channel coefficients as fig. 3.

Step5: Initialize $\mathrm{k}$ and calculate $\mathrm{m}$ using eqn 13. 16.

Step6: Generate the channel using eqn 14, eqn 15 and eqn

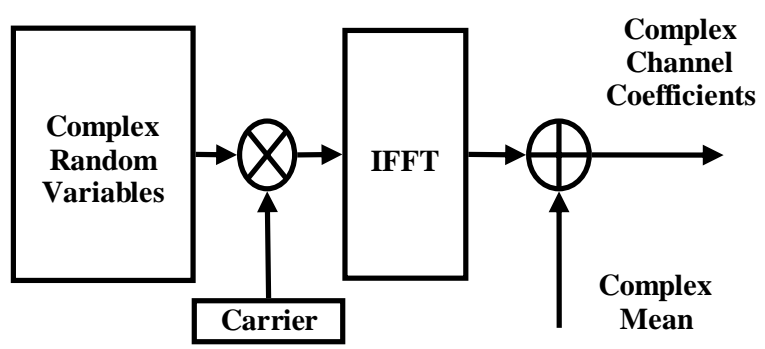

Fig. 3. Generation of the Channel Coefficients

\section{RESUlTS ANALYSIS}

We have simulated Rayleigh, Rician and Nakagami-m fading channel. There is a relationship between the fading factors ' $\mathrm{K}$ ' and ' $\mathrm{m}$ ' as shown in the eqn 13 , so we have initialize ' $\mathrm{K}$ ' and evaluated ' $\mathrm{m}$ ' the fading factor of Nakagami$\mathrm{m}$ fading channel. The simulation is done using Matlab $\mathrm{R} 2013 \mathrm{~b}$ and the results are shown for various value of the fading parameter m. Figure 4 shows the Nakagami-m distribution for same value of scaling parameter $\Omega$ and various value of the fading parameter $\mathrm{m}$. Figure 5 shows the effect of the scaling parameter $\Omega$, keeping the fading parameter $\mathrm{m}$ as constant. In the fig. 6 and 7 the simulated Rayleigh and Rician fading channel were simulated for $\mathrm{m}=1$. As per the eqn. 10, for $\mathrm{m}=1$, the Nakagami- $\mathrm{m}$ distribution converts into a Rayleigh distribution, and the Rician distribution is merely an impulse which could be verified by fig.7 as shown. In the fig.8, the simulated channel is compared with the theoretical channel for $\mathrm{m}=1$. In the subsequent figures, we have shown the Rayleigh, Rician and Nakagami-m channel for various values of $m$ as shown in the figure. It is interesting to know that when the value of $m>1$, the simulated Nakagami-m channel follows Rayleigh fading distribution which could be verified by our simulation results. The channel coefficients are complex values having a real part and imaginary part and it is plotted for absolute amplitude against 1000 samples and is shown in the fig.21. In this work, we have also find out the impulse response of the channel by giving the channel input as 000000000010000000000 and 00000100000 as shown by fig. 23 and fig. 22 respectively.

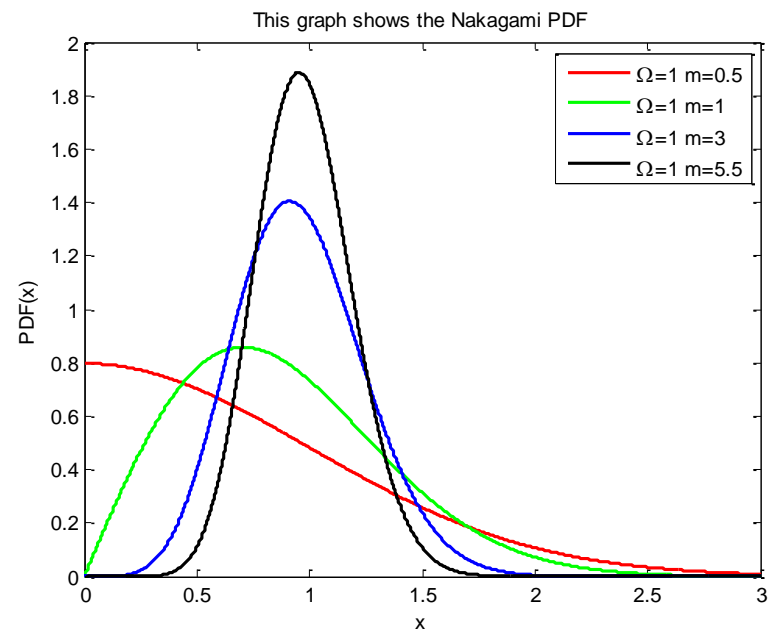

Fig. 4. Nakagami-m distribution plot for $\Omega=1$ and $m=0.5,1,3,5.5$

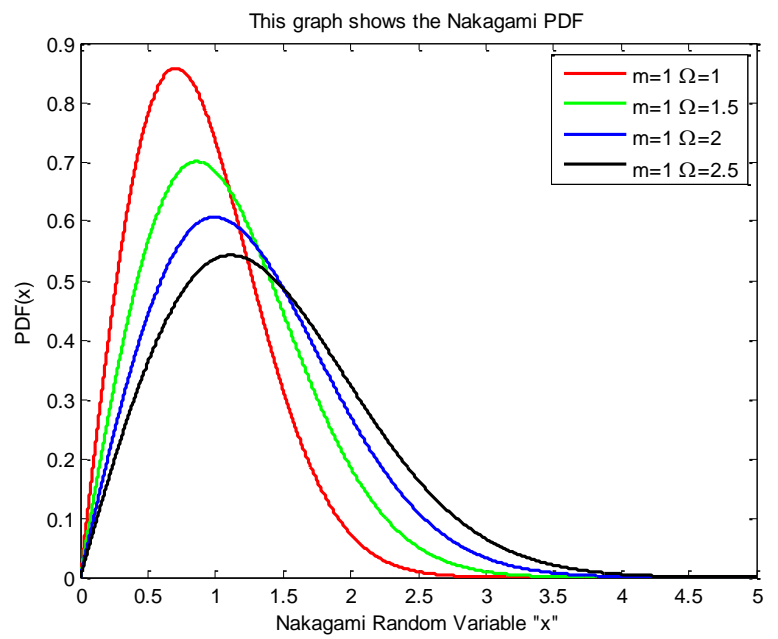

Fig. 5. Nakagami-m distribution plot for $\mathrm{m}=1$ and $\Omega=1,1.5,2,2.5$

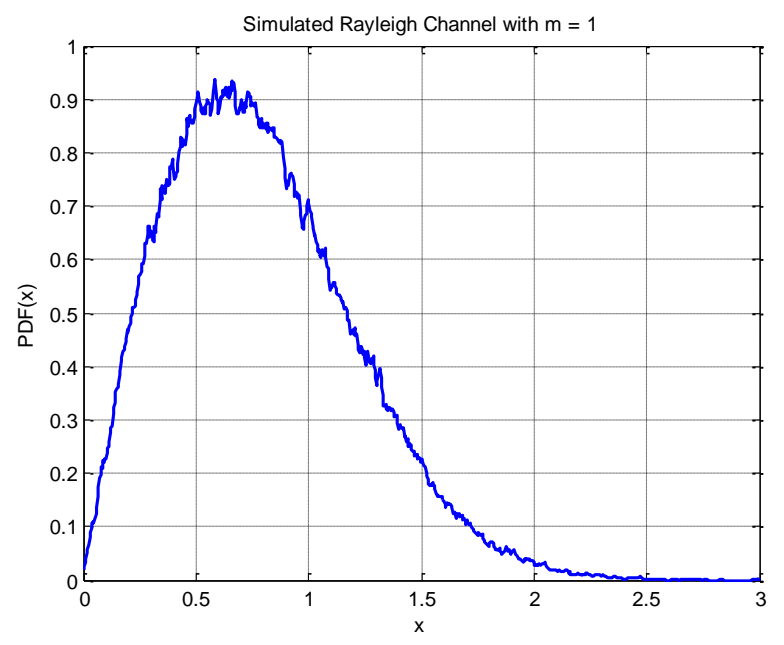

Fig. 6. Simulated Rayleigh Fading Channel with $\mathrm{m}=1$ 


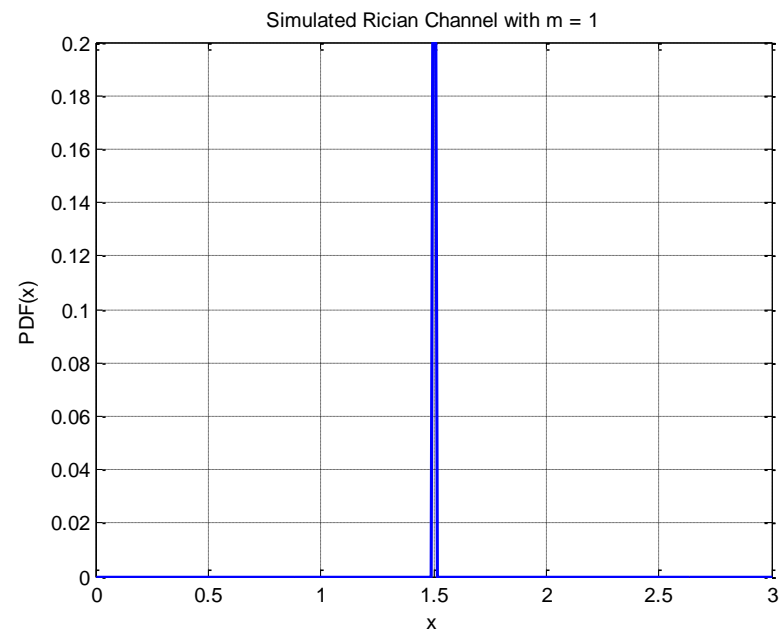

Fig. 7. Simulated Rician Fading Channel with $m=1$

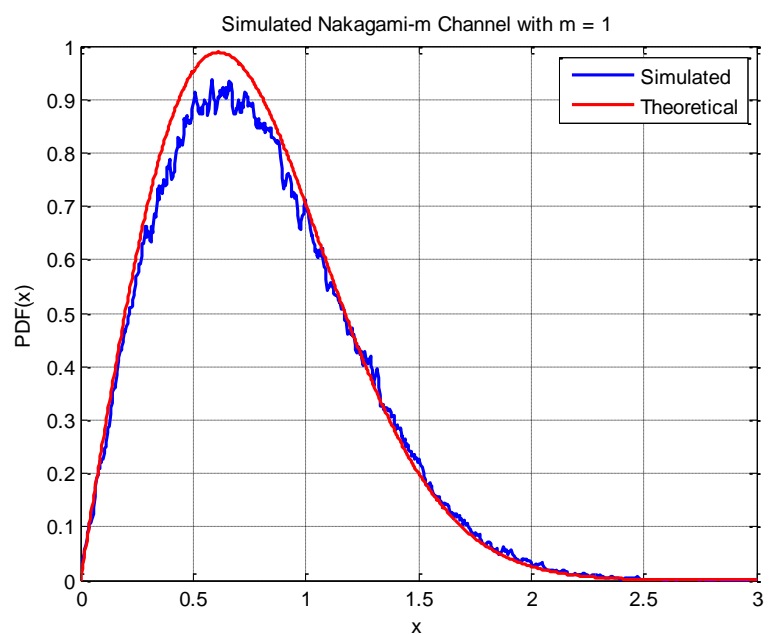

Fig. 8. Simulated Nakagami-m Fading Channel with $m=1$

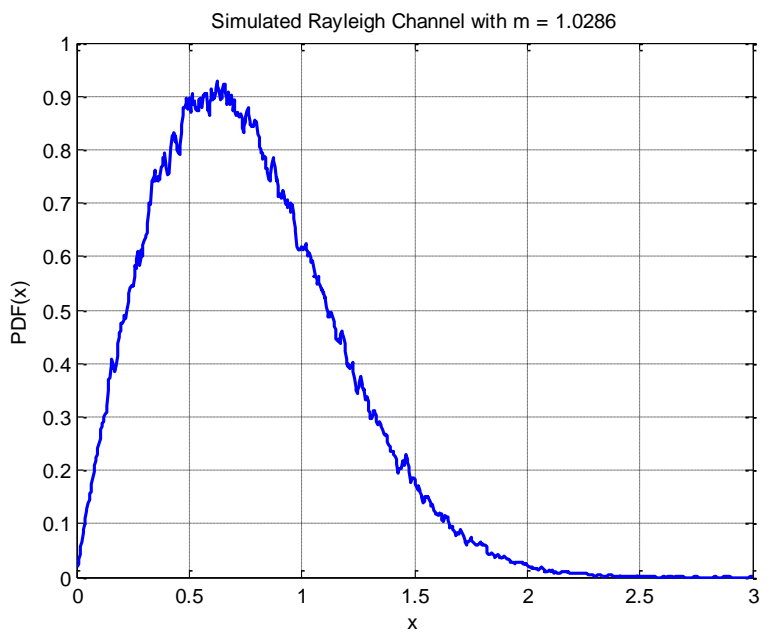

Fig. 9. Simulated Rayleigh Fading Channel with $\mathrm{m}=1.0286$

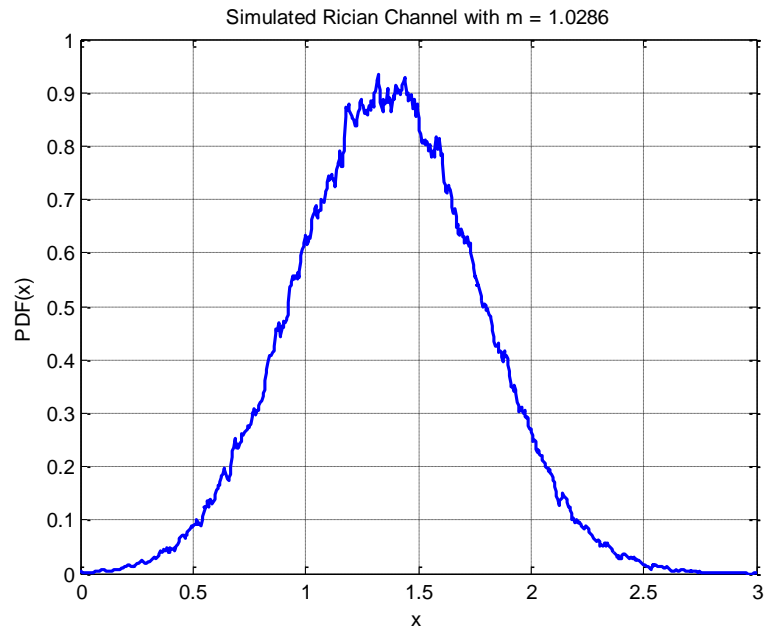

Fig. 10. Simulated Rician Fading Channel with $\mathrm{m}=1.0286$

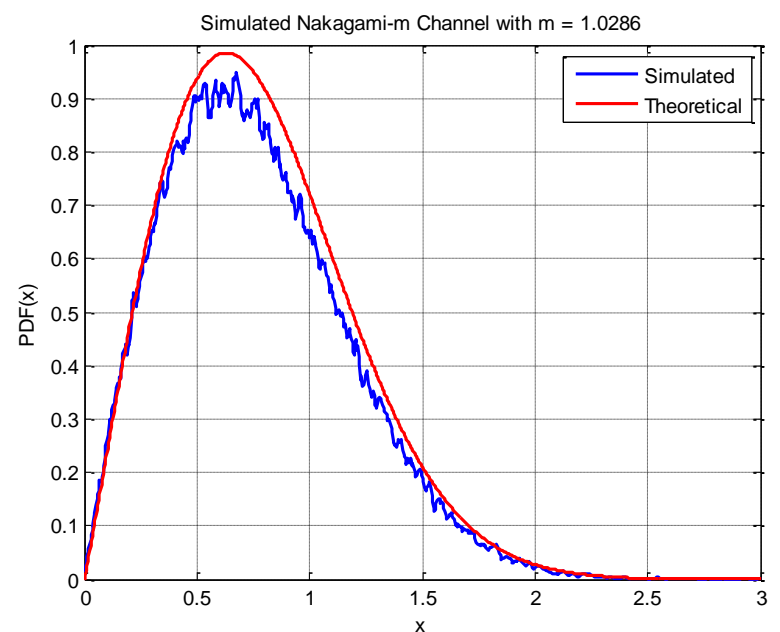

Fig. 11. Simulated Nakagami-m Fading Channel with $m=1.0286$

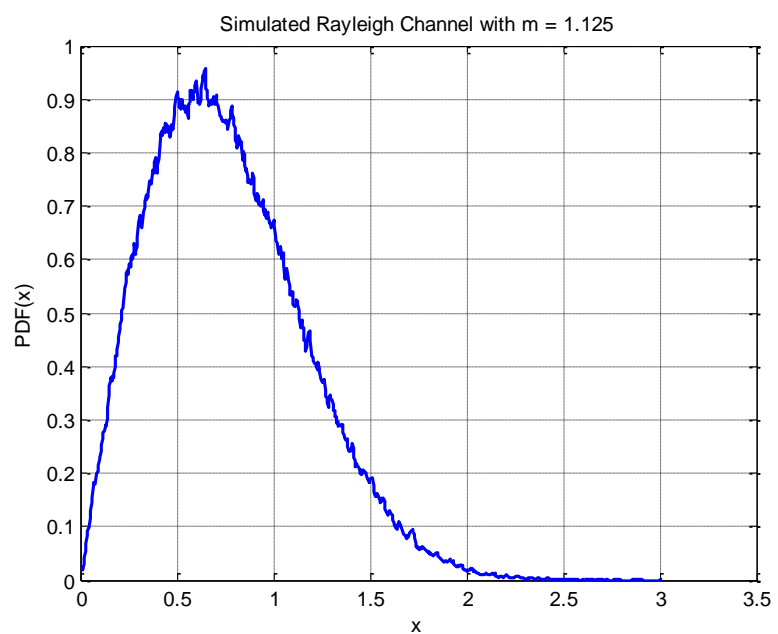

Fig. 12. Simulated Rayleigh Fading Channel with $m=1.125$ 


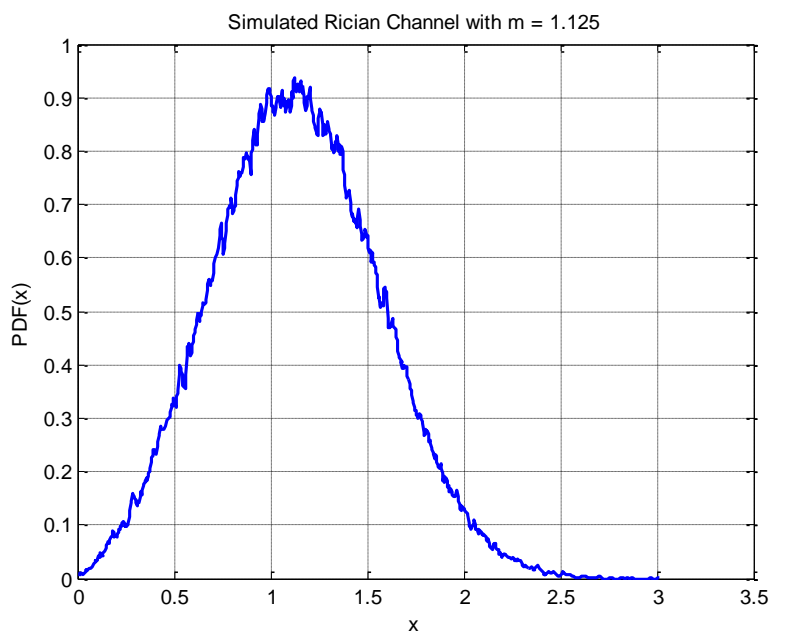

Fig. 13. Simulated Rician Fading Channel with $m=1.125$

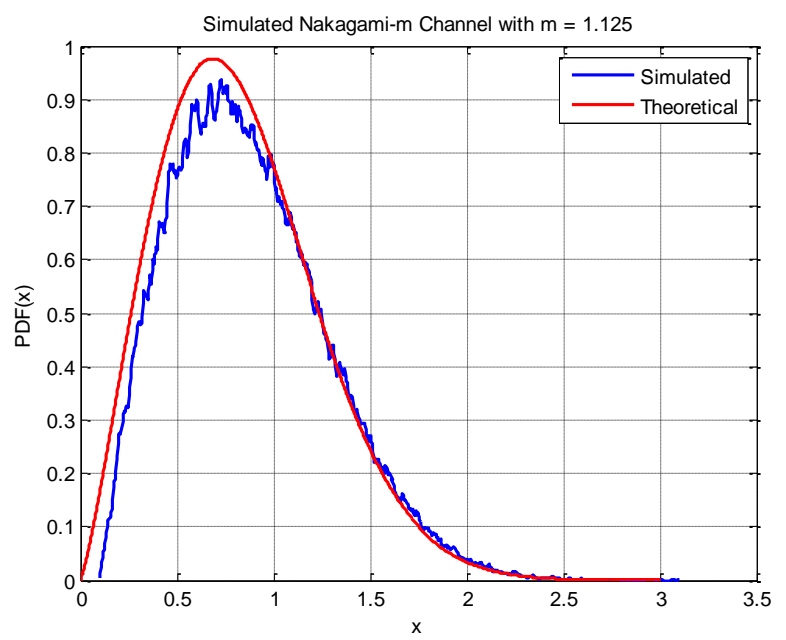

Fig. 14. Simulated Nakagami-m Fading Channel with $m=1.125$

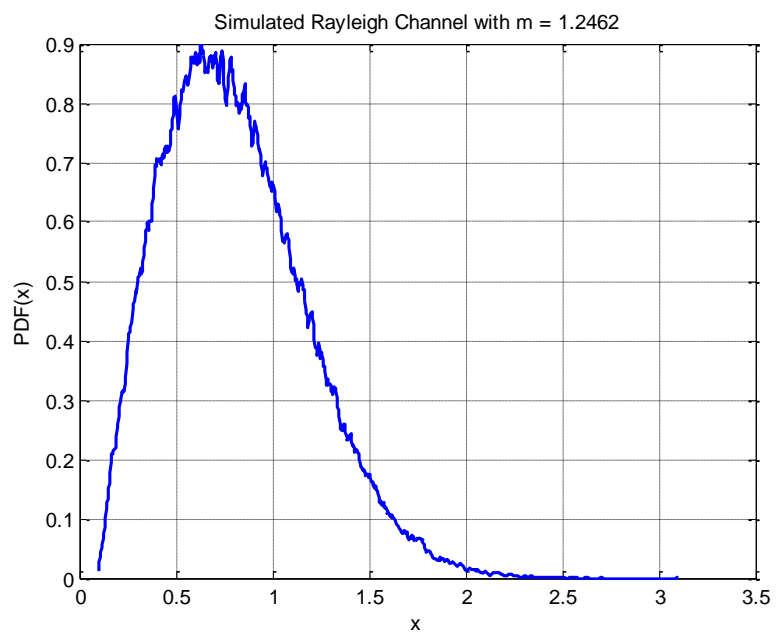

Fig. 15. Simulated Rayleigh Fading Channel with $\mathrm{m}=1.2462$

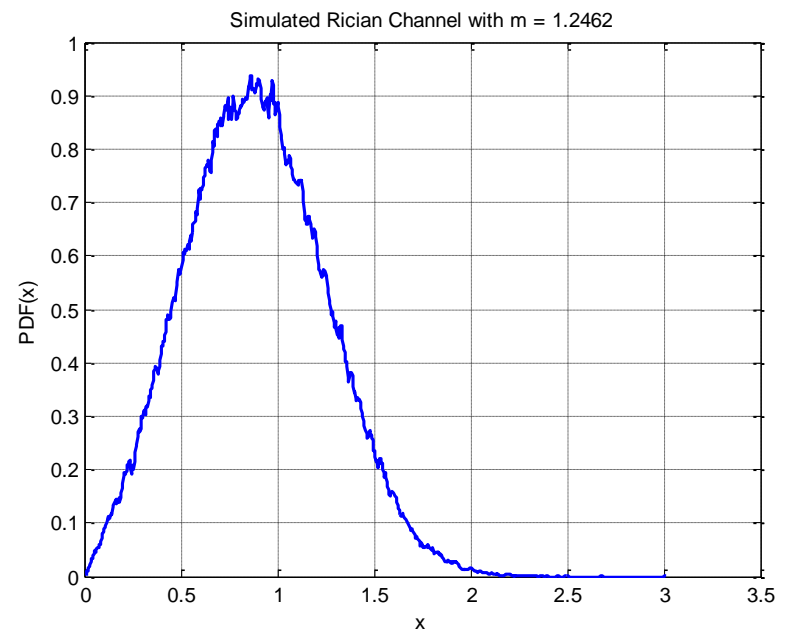

Fig. 16. Simulated Rician Fading Channel with $\mathrm{m}=1.2462$

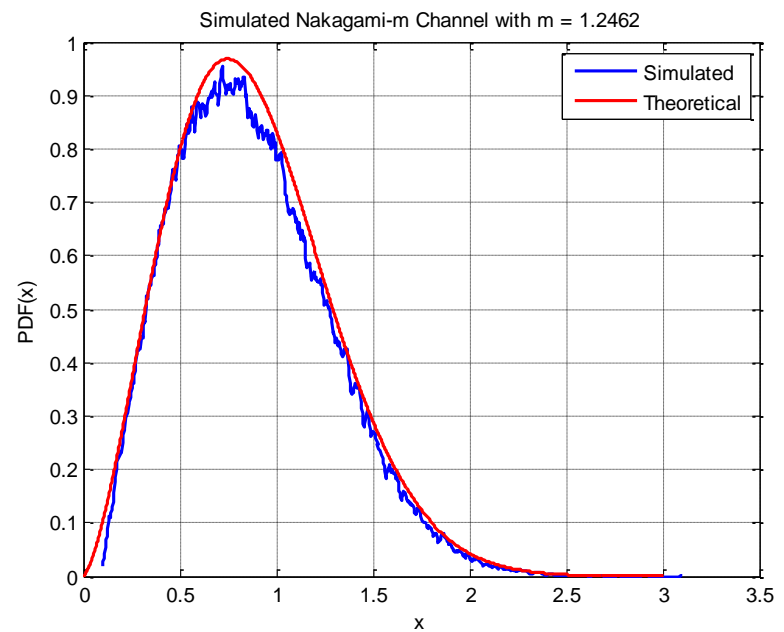

Fig. 17. Simulated Nakagami-m Fading Channel with $m=1.2462$

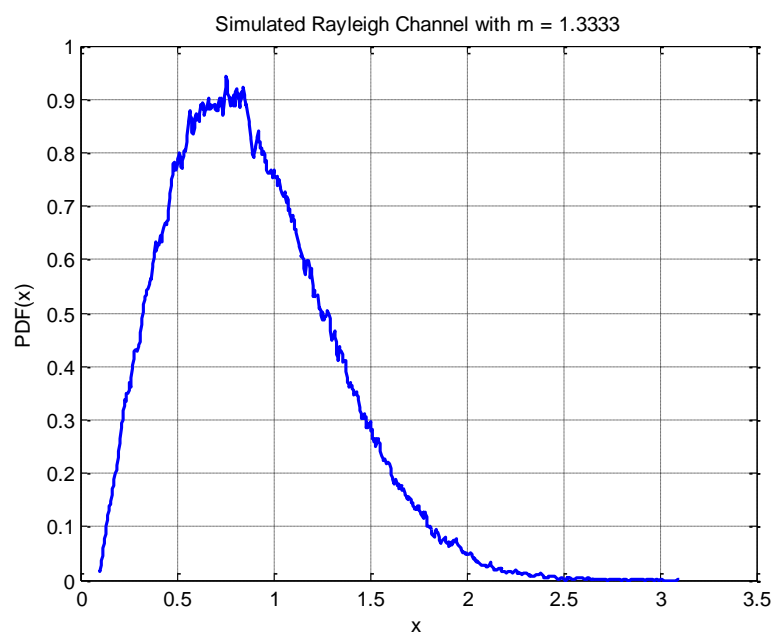

Fig. 18. Simulated Rayleigh Fading Channel with $m=1.3333$ 


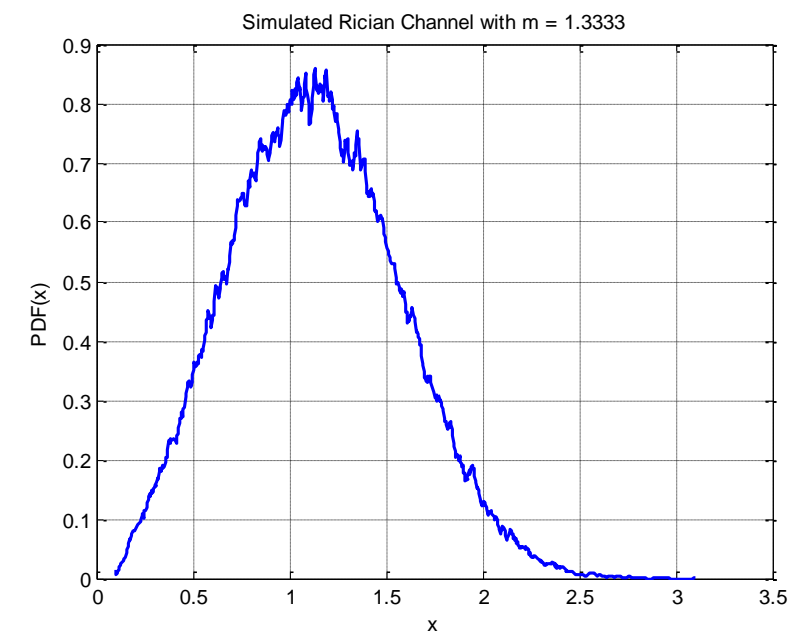

Fig. 19. Simulated Rician Fading Channel with $\mathrm{m}=1.3333$

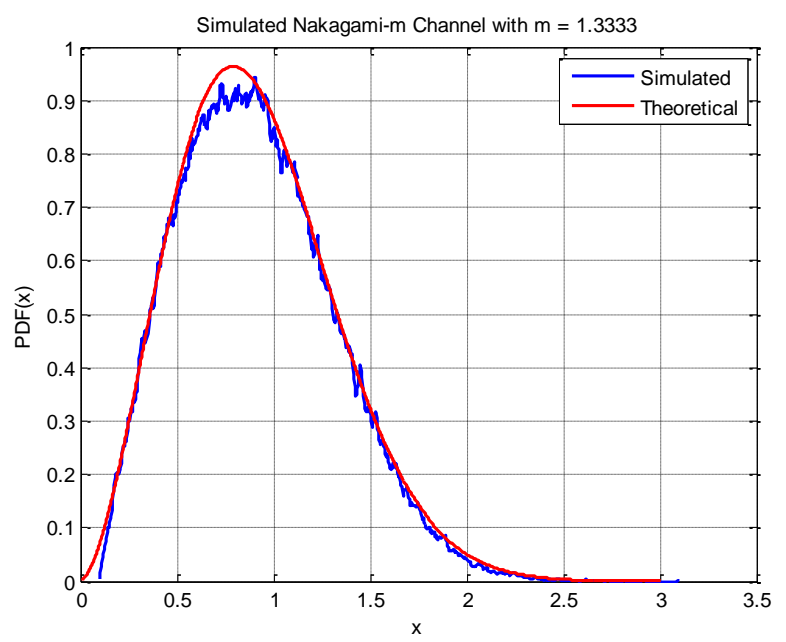

Fig. 20. Simulated Nakagami-m Fading Channel with $m=1.3333$

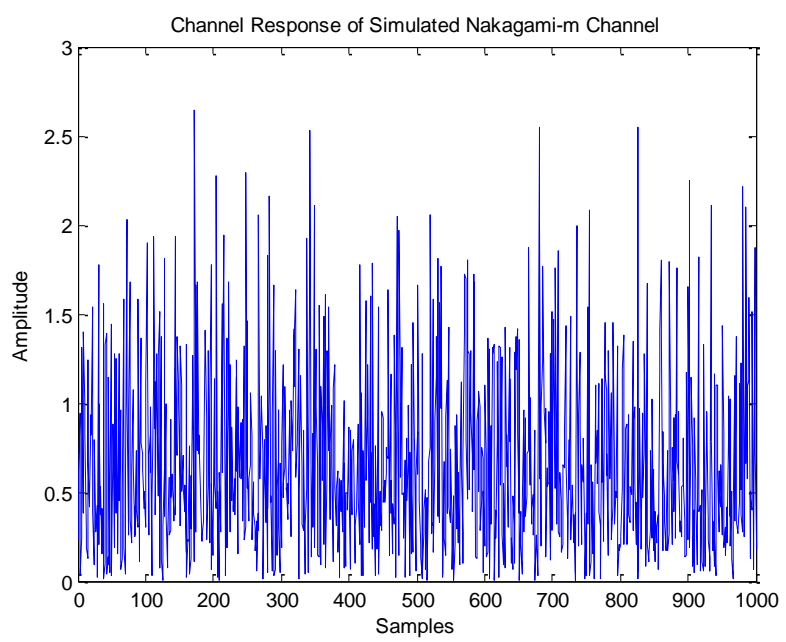

Fig. 21. Channel Response of the Simulated Nakagami-m Fading Channel

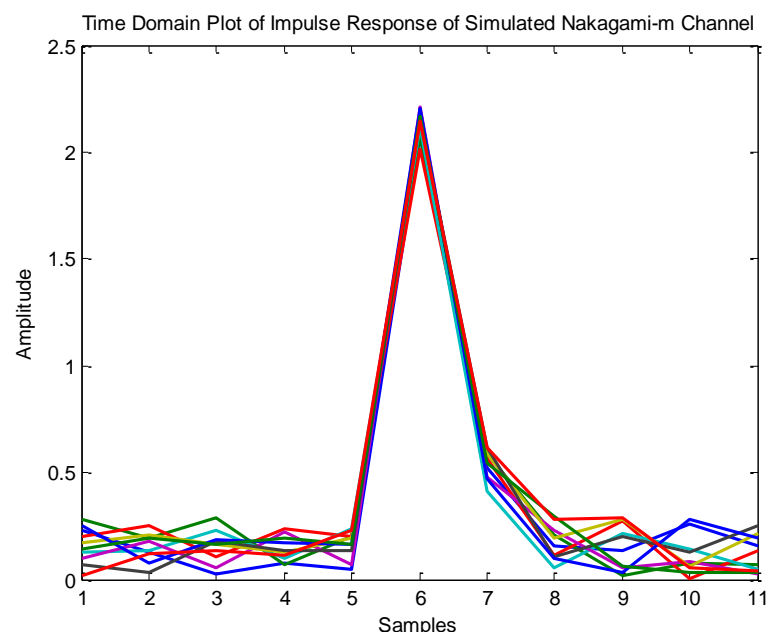

Fig. 22. Impulse Response of the Simulated Nakagami-m Fading Channel when channel input is 00000100000

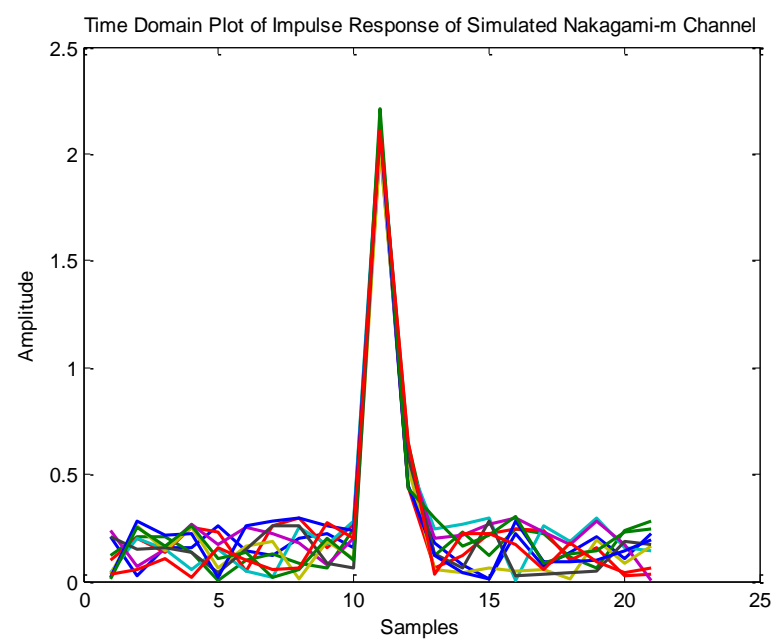

Fig. 23. Impulse Response of the Simulated Nakagami-m Fading Channel when channel input is 000000000010000000000

\section{CONCLUSIONS AND FUTURE WORK}

In this paper, we have discussed the theory needed for the simulation of Nakagami-m fading channel, and propose a statistical model to simulate the same. Rayleigh, Rician and Nakagmai- $m$ fading channels have been simulated and the result of Nakagmi-m channel simulated with the theoretical. In this work, channel response and channel impulse response were also calculated. In future, Monte-Carlo simulation could be applied to know the standard deviation of channel coefficients thus generated over a number of iteration.

\section{REFERENCES}

[1] M. Nakagami. The $m$-distribution - A General Formula of Intensity Distribution of Rapid Fading.In W. C. Hoffman: Statistical Methods of Radio Wave Propagation, Oxford, England, 1960.

[2] M.K. Simon, J.K. Omura, R.A. Scholtz, and B.K. Levitt. Spread Spectrum Communication Handbook. McGraw-Hill Inc, New York, revised edition, 2002. 
[3] A. Lakhzouri, E. S. Lohan, I. Saastamoinen, and M. Renfors "Measurement and Characterization of Satellite-to-Indoor RadioWave Propagation Channel". In CDROM Proc. of The European Navigation Conference (ENC-GNSS '05), Munich, Germany, Jul 2005.

[4] A. Lakhzouri, E. S. Lohan, I. Saastamoinen, and M. Renfors. "Interference and Indoor ChannelPropagation Modeling Based on GPS Satellite Signal Measurements”. In Proc. of ION GPS, pages 896-901, Sep 2005.

[5] M. D. Yacoub, G. Fraidenraich, and J. C. S. Santos Filho, "Nakagami-m phase-envelope joint distribution," Electron. Lett., vol. 41, no. 5, pp.259-261, Mar. 2005.

[6] A.F. Abouraddy and S.M. Elnoubi. "Statistical Modelling of the Indoor Radio Channel at $10 \mathrm{GHz}$ through Propagation Measurements - Part 1: Narrow-band Measurements and Modelling". IEEE Trans. on Vehicular Technology, 49(5):1491-1507, Sep 2000.

[7] M.K. Simon and M.S. Alouini. Digital Communications over Fading Channels: A Unified Approach to Performance Analysis. Wiley InterScience, Sep 2000.

[8] N. C. Beaulieu and C. Cheng. "An Efficient Procedure for Nakagami-m Fading Simulation". In IEEE Proc. of Globecom 2001, volume 6, pages 3336-3342, Nov 2001.

[9] K. W. Yip and T. S. Ng.,'A simulation model for nakagami-m fading channels, $\mathrm{m}<1$ ". IEEETransactions on Communications, 48(2):pp. 214-221, Feb 2000.

[10] L. Schumacher, J. P. Kermoal, F. Frederiksen, K. I. Pedersen, A. Algans, and P. Mogensen. MIMO Channel Characterisation. IST Project IST-1999-11729 METRA Deliverable D2, Feb 2001.
[11] K.I. Pedersen, J. B. Andersen, J. P. Kermoal, and P. Mogensen. "A stochastic multiple-input-multipleoutput radio channel model for evaluation of space-time coding algorithms". In Proc. of IEEE VTC Fall, volume 2, pages 893-897, Boston, USA, Sep 2000.

[12] L. Schumacher, K. I. Pedersen, and P. E. Mogensen. "From antenna spacings to theoretical capacities-guidelines for simulating MIMO systems" . In 13th IEEE International Symposium on Personal, Indoor and Mobile Radio Communications, volume 2, pages 587-592, Sep 2002.

[13] K.N. Nagesh, D. Satyanarayana, S.M.Prabhu, " Statistical analysis of MIMO scheme under Nakagami fading channels",in the proc. of IEEE 16th International Conference on Advanced Communication Technology(ICACT),pp.255 -259, 2014.

[14] Hussain, S.; Fernando, X.N, "Performance Analysis of Relay-Based Cooperative Spectrum Sensing in Cognitive Radio Networks Over NonIdentical Nakagami- m Channels", IEEE Transactions on Communications, vol.62, issue 8, pp.2733 - 2746, 2014.

[15] JianxiaLuo; Zeidler,J.R. "A statistical simulation model for correlated Nakagami fading channels", in the proc. of IEEE International Conference on Communication Technology Proceedings, 2000. WCC - ICCT 2000, vol.2, pp.1680 - 1684.

[16] H. Suzuki, "A statistical model for urban radio propagation," IEEE Trans.on Commun., vol. COM-25, pp. 673-680, July 1977.

[17] U. Charash, "Reception through Nakagami fading multipath channels with random delays," fEEE Trans. Commun., vol. COM-27, pp. 657470, Apr. 1979.

[18] S . Stein, "Fading channel issues in system engineering," IEEEJ. Selecr. Areas Commun., vol. SAC-5, pp. 6849, Feb. 1987. 